\title{
Belief Propagation Based Decoding of Large Non-Orthogonal STBCs
}

\author{
Madhekar Suneel, Pritam Som, A. Chockalingam, and B. Sundar Rajan \\ Department of ECE, Indian Institute of Science, Bangalore 560012, INDIA
}

\begin{abstract}
In this paper, we present a belief propagation (BP) based algorithm for decoding non-orthogonal space-time block codes (STBC) from cyclic division algebras (CDA) having large dimensions. The proposed approach involves message passing on Markov random field (MRF) representation of the STBC MIMO system. Adoption of BP approach to decode non-orthogonal STBCs of large dimensions has not been reported so far. Our simulation results show that the proposed BP-based decoding achieves increasingly closer to SISO AWGN performance for increased number of dimensions. In addition, it also achieves near-capacity turbo coded BER performance; for e.g., with BP decoding of $24 \times 24$ STBC from CDA using BPSK (i.e., 576 real dimensions) and rate-1/2 turbo code (i.e., 12 bps/Hz spectral efficiency), coded BER performance close to within just about 2.5 dB from the theoretical MIMO capacity is achieved.
\end{abstract}

Keywords - Non-orthogonal STBCs, large dimensions, low-complexity decoding, belief propagation, Markov random fields, high spectral efficiencies.

\section{INTRODUCTION}

Use of multiple antennas at the transmitter can offer the benefits of transmit diversity (e.g., using space-time coding) and high data rates (e.g., using spatial multiplexing) [1]. MIMO systems that employ non-orthogonal space-time block codes (STBC) from cyclic division algebras (CDA) for arbitrary number of transmit antennas, $N_{t}$, are particularly attractive because they can simultaneously provide both full-rate (i.e., $N_{t}$ complex symbols per channel use, which is same as in VBLAST) as well as full transmit diversity [2],[3]. The $2 \times 2$ Golden code is a well known non-orthogonal STBC from CDA for 2 transmit antennas [4]. High spectral efficiencies of the order of tens of bps/Hz can be achieved using large non-orthogonal STBCs. For e.g., a $16 \times 16$ STBC from CDA has 256 complex symbols in it with 512 real dimensions; with 16-QAM and rate-3/4 turbo code, this system offers a high spectral efficiency of $48 \mathrm{bps} / \mathrm{Hz}$. Decoding of non-orthogonal STBCs with such large dimensions, however, has been a challenge. Sphere decoder and its low-complexity variants are prohibitively complex for decoding such STBCs with hundreds of dimensions. Recently, we proposed a low-complexity near-ML achieving algorithm to decode large non-orthogonal STBCs from CDA; this algorithm, which is based on bitflipping approach, is termed as likelihood ascent search (LAS) algorithm [5]-[7]. Our new contribution in this paper is that we present a belief propagation $(B P)$ based approach to decoding of non-orthogonal STBCs with large dimensions, and report very good uncoded BER and near-capacity performance. To our knowledge, BP has not been reported for decoding non-orthogonal STBCs from CDA having large dimensions.

Belief propagation [8] is known to be well suited in several communication problems [9]; e.g., decoding of turbo codes and LDPC codes [10], [11], multiuser detection [12],[13], signal detection in ISI channels [14],[15], and MIMO detection [16],[17]. Taking the cue from the success of BP in decoding turbo codes and achieving near-capacity performance with large frame sizes, in this paper we achieve near-ML and nearcapacity performance in decoding STBCs with large dimensions using BP at practical complexities. It is known that graphical models that represent MIMO systems are highly connected. While BP was initially formalized for loop-free graphs, it has been empirically found to work in loopy graphs as well [10], [18]. In the context of MIMO detection using $\mathrm{BP}$, [16] reported a successful adoption of BP algorithm on Markov random fields (MRF) by employing belief propagation meant for pairwise MRFs as described in [8]. It presented the BER performance of the BP based detector for VBLAST with $N_{t}=4$ and $N_{r}=4,6,8$ without and with simulated annealing. However, BP approach applied to large dimension decoding in MIMO systems, particularly large nonorthogonal STBC MIMO systems, has not been reported so far. In this regard, our work here is the first in reporting $\mathrm{BP}$ on MRFs for large dimension STBC MIMO decoding. Our simulation results show that the proposed BP decoding algorithm performs close to within just about $2.5 \mathrm{~dB}$ from the theoretical MIMO capacity in a $24 \times 24$ non-orthogonal STBC MIMO system using BPSK and rate-1/2 turbo code at a spectral efficiency of $12 \mathrm{bps} / \mathrm{Hz}$. We also present the performance of BP decoding in the presence of spatial correlation. We show that the loss in performance due to spatial correlation can be alleviated by using increased receive spatial dimensions.

\section{Non-Orthogonal StBC MIMO System Model}

Consider a STBC MIMO system with multiple transmit and multiple receive antennas. An $(n, p, k) \mathrm{STBC}$ is represented by a matrix $\mathbf{X}_{c} \in \mathbb{C}^{n \times p}$, where $n$ and $p$ denote the number of transmit antennas and number of time slots, respectively, and $k$ denotes the number of complex data symbols sent in one STBC matrix. The $(i, j)$ th entry in $\mathbf{X}_{c}$ represents the complex number transmitted from the $i$ th transmit antenna in the $j$ th time slot. The rate of an STBC is $\frac{k}{p}$. Let $N_{r}$ and $N_{t}=n$ denote the number of receive and transmit antennas, respectively. Let $\mathbf{H}_{c} \in \mathbb{C}^{N_{r} \times N_{t}}$ denote the channel gain matrix, where the $(i, j)$ th entry in $\mathbf{H}_{c}$ is the complex channel gain from the $j$ th transmit antenna to the $i$ th receive antenna. We assume that the channel gains remain constant over one STBC matrix duration. Assuming rich scattering, we model the entries of $\mathbf{H}_{c}$ as i.i.d $\mathcal{C N}(0,1)$. The received space-time signal matrix, $\mathbf{Y}_{c} \in \mathbb{C}^{N_{r} \times p}$, can be written as

$$
\mathbf{Y}_{c}=\mathbf{H}_{c} \mathbf{X}_{c}+\mathbf{N}_{c}
$$

where $\mathbf{N}_{c} \in \mathbb{C}^{N_{r} \times p}$ is the noise matrix at the receiver and its entries are modeled as i.i.d $\mathcal{C N}\left(0, \sigma^{2}=\frac{N_{t} E_{s}}{\gamma}\right)$, where $E_{s}$ is the average energy of the transmitted symbols, and $\gamma$ is the average received SNR per receive antenna [1], and the $(i, j)$ th entry in $\mathbf{Y}_{c}$ is the received signal at the $i$ th receive antenna in the $j$ th time slot. Consider linear dispersion STBCs, where $\mathbf{X}_{c}$ can be written in the form [1] 


$$
\mathbf{X}_{c}=\sum_{i=1}^{k} x_{c}^{(i)} \mathbf{A}_{c}^{(i)}
$$

where $x_{c}^{(i)}$ is the $i$ th complex data symbol, and $\mathbf{A}_{c}^{(i)} \in \mathbb{C}^{N_{t} \times p}$ is its weight matrix. The received signal model in (1) can be written in an equivalent V-BLAST form as

$$
\mathbf{y}_{c}=\sum_{i=1}^{k} x_{c}^{(i)}\left(\widehat{\mathbf{H}}_{c} \mathbf{a}_{c}^{(i)}\right)+\mathbf{n}_{c}=\widetilde{\mathbf{H}}_{c} \mathbf{x}_{c}+\mathbf{n}_{c}
$$

where $\mathbf{y}_{c} \in \mathbb{C}^{N_{r} p \times 1}=\operatorname{vec}\left(\mathbf{Y}_{c}\right), \widehat{\mathbf{H}}_{c} \in \mathbb{C}^{N_{r} p \times N_{t} p}=(\mathbf{I} \otimes$ $\left.\mathbf{H}_{c}\right), \mathbf{a}_{c}^{(i)} \in \mathbb{C}^{N_{t} p \times 1}=\operatorname{vec}\left(\mathbf{A}_{c}^{(i)}\right), \mathbf{n}_{c} \in \mathbb{C}^{N_{r} p \times 1}=\operatorname{vec}\left(\mathbf{N}_{c}\right)$, $\mathbf{x}_{c} \in \mathbb{C}^{k \times 1}$ whose $i$ th entry is the data symbol $x_{c}^{(i)}$, and $\widetilde{\mathbf{H}}_{c} \in$ $\mathbb{C}^{N_{r} p \times k}$ whose $i$ th column is $\widehat{\mathbf{H}}_{c} \mathbf{a}_{c}^{(i)}, i=1,2, \cdots, k$. For notational simplicity, we drop the subscripts $c$ in (3) and write

$$
\mathbf{y}=\mathbf{H x}+\mathbf{n},
$$

where $\mathbf{x}=\mathbf{x}_{c}, \mathbf{H}=\widetilde{\mathbf{H}}, \mathbf{y}=\mathbf{y}_{c}$, and $\mathbf{n}=\mathbf{n}_{c}$. We note that (4) can be viewed as an equivalent V-BLAST representation of the non-orthogonal STBC MIMO system. We assume that the channel coefficients are known at the receiver but not at the transmitter.

\section{A. High-Rate Non-Orthogonal STBCs from CDA}

We consider square (i.e., $n=p=N_{t}$ ), full-rate (i.e., $k=p n=$ $N_{t}^{2}$ ), circulant (where the weight matrices $\mathbf{A}_{c}^{(i)}$,s are permutation type), non-orthogonal STBCs from CDA [2], whose construction for arbitrary number of transmit antennas $n$ is given by the matrix in (4.a) given at the bottom of this page [2]. In (4.a), $\omega_{n}=e^{\frac{\mathbf{j} 2 \pi}{n}}, \mathbf{j}=\sqrt{-1}$, and $d_{u, v}, 0 \leq u, v \leq n-1$ are the $n^{2}$ data symbols from a QAM alphabet. When $\delta=$ $t=1$, the code in (4.a) is information lossless (ILL), and when $\delta=e^{\sqrt{5} \mathbf{j}}$ and $t=e^{\mathbf{j}}$, it is of full-diversity and information lossless (FD-ILL) [2]. High spectral efficiencies with large $N_{t}$ can be achieved using this code construction; e.g., $16 \times 16 \mathrm{STBC}$ from (4.a) using 4-QAM and rate-3/4 turbo code offers a spectral efficiency of $24 \mathrm{bps} / \mathrm{Hz}$ along with the full-diversity of order $N_{t} N_{r}$ under ML detection. However, since these STBCs are non-orthogonal, ML detection gets increasingly impractical for large $N_{t}$. Hence, a key challenge in realizing the benefits of these large non-orthogonal STBCs in practice is that of achieving near-ML performance for large $N_{t}$ at low decoding complexities. The BP based decoding approach in this paper essentially addresses this issue.

\section{BP ON MRFS FOR LARGE DIMENSION MIMO DETECTION/DECODING}

In this section, we present the BP detection algorithm assuming a V-BLAST system with BPSK modulation. The algorithm can be applied on the equivalent V-BLAST representation of the non-orthogonal STBC MIMO system given by (4). $\mathrm{BP}$ is a technique that solves inference problems using graphical models such as factor graphs, Bayesian belief networks

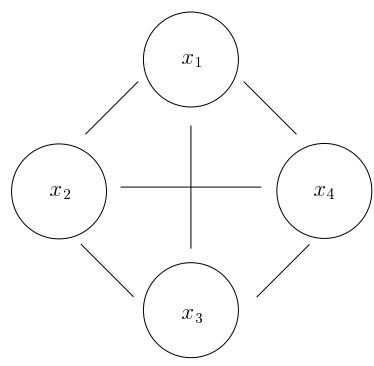

Fig. 1

MARKOV RANDOM FIELD REPRESENTATION OF A BPSK V-BLAST SYSTEM WITH 4 TRANSMIT ANTENNAS.

and MRFs. In this paper, we consider BP on MRF representation of MIMO systems.

\section{A. MRF Representation of a MIMO System}

MRFs are graphs that indicate inter-dependencies between random variables [9]. An MRF is an undirected graph whose vertices are random variables. The variables are such that any variable is independent of all the other variables, given its neighbors, i.e.,

$$
\mathrm{p}\left(x_{k} \mid x_{1}, \ldots, x_{k-1}, x_{k+1}, \ldots, x_{N}\right)=\mathrm{p}\left[x_{k} \mid \mathcal{N}\left(x_{k}\right)\right],
$$

where $\mathcal{N}\left(x_{k}\right)$ represents the set of all nodes neighboring the node pertaining to the variable $x_{k}$. Usually, the variables in an MRF are constrained by a compatibility function, also known as a clique potential in literature. A clique of an MRF is a fully connected sub-graph that does not remain fully connected if any additional vertex of the MRF is included in it. This is sometimes called a maximal clique, but we shall use the term clique to refer to a maximal clique. Let there be $N_{C}$ cliques in the MRF, and $\mathbf{x}_{j}$ be the set of variables in clique $j$. Let $\psi_{j}\left(\mathbf{x}_{j}\right)$ be the clique potential of clique $j$. Then the joint distribution of the variables is

$$
\mathrm{p}(\mathbf{x}) \propto \prod_{j=1}^{N_{C}} \psi_{j}\left(\mathbf{x}_{j}\right),
$$

where $\mathbf{x}$ is the set of all variables in the graph. For example, consider the MRF representation of a BPSK V-BLAST system with 4 transmit antennas as shown in Fig. 1 Here, $x_{1}, x_{2}, x_{3}, x_{4}$ respectively are the binary symbols transmitted from the four transmit antennas. Each of these symbols assumes a value from $\{ \pm 1\}$. In a V-BLAST system, since every transmitted symbol interferes with every other transmitted symbol at the receiver, the MRF is fully-connected, and contains a single clique, namely, $\left\{x_{1}, x_{2}, x_{3}, x_{4}\right\}$. The joint probability distribution is

$$
\begin{array}{r}
\mathrm{p}\left(x_{1}, x_{2}, x_{3}, x_{4}\right)=\mathrm{p}\left(x_{1}\right) \mathrm{p}\left(x_{2} \mid x_{1}\right) \mathrm{p}\left(x_{3} \mid x_{1}, x_{2}\right) \\
\mathrm{p}\left(x_{4} \mid x_{1}, x_{2}, x_{3}\right) .
\end{array}
$$

B. Pairwise $M R F_{S}$

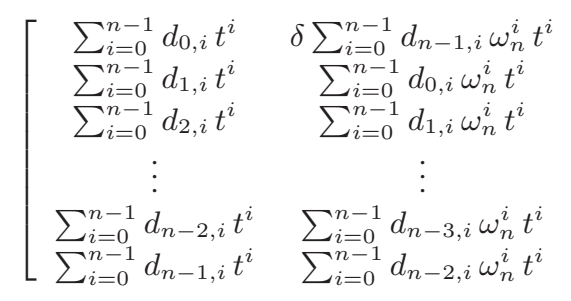

$\delta \sum_{i=0}^{n-1} d$

$\delta \sum_{i=0}^{n-1} d_{n-1, i} \omega_{n}^{2 i} t^{i} \ldots$

$\sum_{i=0}^{n-1} d_{0, i} \omega_{n}^{2 i} t^{i} \quad \cdots$

$\vdots$
$\sum_{i=0}^{n-1} d_{n-4, i} \omega_{n}^{2 i} t^{i}$

$\sum_{i=0}^{n-1} d_{n-3, i} \omega_{n}^{2 i} t^{i}$

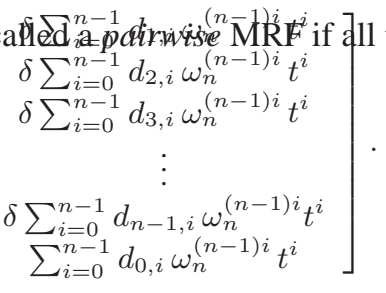




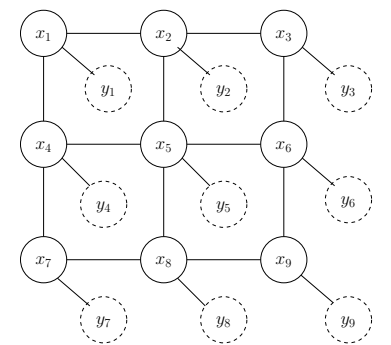

Fig. 2

AN EXAMPLE OF A PAIRWISE MARKOV RANDOM FIELD WITH OBSERVED (EXPLICIT) VARIABLES AND HIDDEN (IMPLICIT) VARIABLES.

MRF are of size two. In this case, the clique potentials are all functions of two variables. The clique potentials can then be denoted as $\psi_{i, j}\left(x_{i}, x_{j}\right)$, where $x_{i}, x_{j}$ are variables connected by an edge in the MRF.

Consider a pairwise MRF in which the $x_{i}$ 's denote underlying hidden variables on which the observed variables $y_{i}$ 's are dependent [8]. Let the dependence between the hidden variable $x_{i}$ and the explicit variable $y_{i}$ be represented by a joint compatibility function $\phi_{i}\left(x_{i}, y_{i}\right)$. This scenario is shown in Fig. 2. In such a scenario, the joint distribution of the hidden and explicit variables is

$$
\mathrm{p}(\mathbf{x}, \mathbf{y}) \propto \prod_{i, j} \psi_{i, j}\left(x_{i}, x_{j}\right) \prod_{i} \phi_{i}\left(x_{i}, y_{i}\right) .
$$

\section{Belief Propagation on Pairwise MRFs}

Consider a situation similar to that of Fig. 2 where $x_{i}$ 's are the hidden variables and $y_{i}$ 's are the observed variables. If we consider the $y_{i}$ 's to be fixed and write $\phi_{i}\left(x_{i}, y_{i}\right)$ simply as $\phi_{i}\left(x_{i}\right)$, then, from (8), the joint distribution for the hidden variables can be written as [8]

$$
\mathrm{p}(\mathrm{x}) \propto \prod_{i, j} \psi_{i, j}\left(x_{i}, x_{j}\right) \prod_{i} \phi_{i}\left(x_{i}\right) .
$$

A message from node $j$ to node $i$ denoted as $m_{j, i}\left(x_{i}\right)$, and belief at node $i$ denoted as $b_{i}\left(x_{i}\right)$ are vectors of length equal to the number of values that the discrete variable $x_{i}$ can possibly take (e.g., length of message and belief vectors is 2 in BPSK since $x_{i} \in\{ \pm 1\}$ ). Each element of the belief vector is proportional to how likely the corresponding value of $x_{i}$ was transmitted. On the other hand, each element in the message vector $m_{j i}\left(x_{i}\right)$ is proportional to how likely $x_{j}$ thinks the corresponding value of $x_{i}$ has been transmitted. The belief at node $i$ about the state of $x_{i}$ is

$$
\mathrm{b}_{i}\left(x_{i}\right) \propto \phi_{i}\left(x_{i}\right) \prod_{j \in \mathcal{N}(i)} m_{j, i}\left(x_{i}\right) .
$$

In particular, the messages are defined as [8]

$$
m_{j, i}\left(x_{i}\right) \propto \sum_{x_{j}} \phi_{j}\left(x_{j}\right) \psi_{j, i}\left(x_{j}, x_{i}\right) \prod_{k \in \mathcal{N}(j) \backslash i} m_{k, j}\left(x_{j}\right)
$$

Equation (11) actually constitutes an iteration, as the message is defined in terms of the other messages. Therefore, belief propagation essentially involves computing the outgoing messages from a node to each of its neighbors using the local joint-compatibility function and the incoming messages and transmitting them.

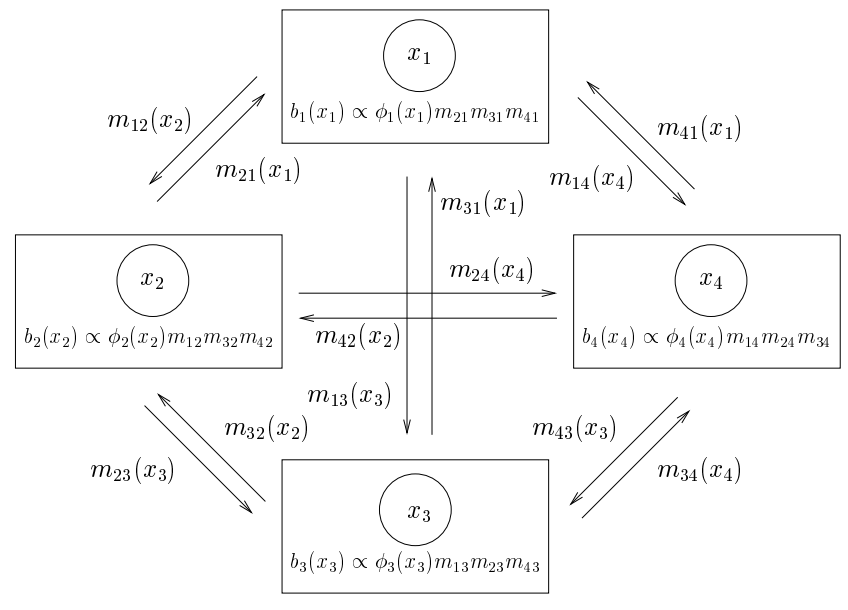

$m_{i j}\left(x_{j}\right)=\sum_{x_{i} \in\{ \pm 1\}} \phi_{i}\left(x_{i}\right) \psi_{i j}\left(x_{i}, x_{j}\right) \prod_{k \in N(i) \backslash j} m_{k i}\left(x_{i}\right) ;$

$N(i)=$ Set of neighbours of $i$ th node.

e.g., $m_{12}\left(x_{2}\right)=\sum_{x_{1} \in\{ \pm 1\}} \phi_{1}\left(x_{1}\right) \psi_{12}\left(x_{1}, x_{2}\right) m_{31}\left(x_{1}\right) m_{41}\left(x_{1}\right)$

Fig. 3

MESSAGE PASSING IN AN MRF FOR A BPSK V-BLAST SYSTEM WITH 4 TRANSMIT ANTENNAS.

\section{BP for MIMO Detection}

In this subsection, for the MIMO system defined by an MRF (e.g., as shown in the graph in Fig. 1), we present the BP based detection algorithm. We observe that the MRFs for MIMO systems, exemplified in Fig. 1, are not pairwise MRFs. However, application of the BP algorithm for pairwise MRFs, as outlined by (10) and 111), to the MRFs of MIMO systems yields a low-complexity detector for MIMO systems with large dimensions. The joint probability distribution for hidden variables $x_{i}$ in a pairwise MRF is given by (9). We use the following functions $\phi_{i}$ and $\psi_{i, j}$ for MIMO detection using BP:

$$
\begin{aligned}
\phi_{i}\left(x_{i}\right) & =\exp \left[\Re\left(x_{i}^{H} z_{i}\right)+\ln \left\{\mathrm{p}\left(x_{i}\right)\right\}\right], \\
\psi_{i, j}\left(x_{i}, x_{j}\right) & =\Re\left(\exp \left(-x_{i}^{H} R_{i, j} x_{j}\right)\right),
\end{aligned}
$$

where [16]

$$
\begin{aligned}
\mathbf{z} & \triangleq \frac{1}{\sigma^{2}} \mathbf{H}^{H} \mathbf{y}, \\
\mathbf{R} & \triangleq \frac{1}{\sigma^{2}} \mathbf{H}^{H} \mathbf{H} .
\end{aligned}
$$

Then, the message from node $i$ to a neighboring node $j$ in the $\mathrm{BP}$ algorithm is given by [11] as given in [8]. For a BPSK VBLAST system with 4 transmit antennas, the message passing scheme is summarized in Fig. 3. Nodes pass messages to each other in an iterative fashion. After the last iteration, beliefs are calculated locally at each node. The value of $x_{i}$ which has the maximum belief is selected as the symbol decision. The belief is the soft-output of the detector which can be fed to the decoder in a coded system.

\section{E. Computational Complexity}

The complexity of the detection scheme comprises of three components, namely, $i$ ) computation of channel correlation

\footnotetext{
${ }^{1}$ Our simulation results show that near SISO AWGN uncoded BER performance and near-capacity coded BER performance are achieved in large dimension V-BLAST as well as non-orthogonal STBC MIMO systems by this approximate approach.
} 
$\mathbf{R}$ given by (15) and matched filter output $\mathbf{z}$ given by (14), ii) computation of local evidence $\phi$ and compatibility function $\psi$ in (12) and (13), respectively, and iii) calculation of beliefs and messages during iterative message passing given by (10) and (11), respectively. The computation of $\mathbf{R}$ and $\mathbf{z}$ involves the computation of $\mathbf{H}^{H} \mathbf{H}$ and $\mathbf{H}^{H} \mathbf{y}$, respectively. In case of STBC MIMO system, two good properties of the STBCs from CDA are instrumental in achieving low orders of complexity for the computation of $\mathbf{H}^{H} \mathbf{H}$ and $\mathbf{H}^{H} \mathbf{y}$. They are: $i$ ) the weight matrices $\mathbf{A}_{c}^{(i)}$,s are permutation type, and ii) the $N_{t}^{2} \times N_{t}^{2}$ matrix formed with $N_{t}^{2} \times 1$-sized $\mathbf{a}_{c}^{(i)}$ vectors as columns is a scaled unitary matrix. For $N_{t}=N_{r}$, the computation of $\mathbf{H}^{H} \mathbf{y}$ and hence $\mathbf{z}$ can be done in $O\left(N_{t}^{4}\right)$ complexity, i.e., in $O\left(N_{t}^{2}\right)$ per-symbol complexity since there are $N_{t}^{2}$ symbols in one STBC matrix. Likewise, the computation of $\mathbf{H}^{H} \mathbf{H}$ and hence $\mathbf{R}$ can be done in $O\left(N_{t}^{3}\right)$ per-symbol complexity. In case of V-BLAST, $\mathbf{R}$ and $\mathbf{z}$ can be computed in $O\left(N_{t}^{2}\right)$ and $O\left(N_{t}\right)$ per-symbol complexity, respectively. Computation of $\psi$ involves $O\left(N_{t}^{2}\right)$ and $O\left(N_{t}\right)$ per-symbol complexity for STBC and V-BLAST, respectively. The persymbol complexity of computing $\phi$ is $O(1)$. The per-symbol complexities involved in the computation of messages and beliefs in a single iteration for V-BLAST are $O\left(N_{t}^{2}\right)$ and $O\left(N_{t}\right)$, respectively; for STBC, these complexities are of or$\operatorname{der} O\left(p^{2} N_{t}^{2}\right)$ and $O\left(p N_{t}\right)$, respectively.

\section{Simulation Results}

Our simulation results have shown that the proposed BP based algorithm achieves increasingly closer to SISO AWGN uncoded BER performance for V-BLAST signals with increasing number of dimensions (e.g., performance close to within $1 \mathrm{~dB}$ of $10^{-3}$ uncoded BER for hundreds of dimensions). Since the dimensions in V-BLAST are in space alone, systems with hundreds of antennas may not be realistic. On the other hand, use of non-orthogonal STBCs from CDA can create hundreds of dimensions with just tens of antennas (space) and tens of channel uses (time). In this section, we present the uncoded and coded BER performance of the proposed BP algorithm in decoding large non-orthogonal STBCs. 5 BP iterations are used in all the simulations.

\section{A. Uncoded BER performance of large STBCs from CDA:}

$B P$ decoding achieves near SISO AWGN performance for large STBCs: In Fig. 4, we plot the uncoded BER performance of $8 \times 8$ (64 dimensions), $16 \times 16$ (256 dimensions) and $24 \times 24$ (576 dimensions) non-orthogonal STBCs from CDA for BPSK and $N_{t}=N_{r}$, as a function of average received SNR per receive antenna, $\gamma$ [1]. BER plots for STBCs with ILL (i.e., $\delta=t=1$ ) and FD-ILL (i.e., $\delta=e^{\sqrt{5} \mathbf{j}}, t=e^{\mathbf{j}}$ ) are shown. For reference purposes, we have plotted the BPSK BER performance on a SISO AWGN channel as well as on a SISO flat-fading channel. From Fig. 4 the following two interesting observations can be made:

- BERs of both ILL and FD-ILL STBCs with BP decoding improve and approach SISO AWGN performance as the number of dimensions (i.e., $N_{t}^{2}$ ) is increased. For e.g., the performance of $24 \times 24$ FD-ILL STBC is just about $1.5 \mathrm{~dB}$ away from SISO AWGN performance at $10^{-3}$

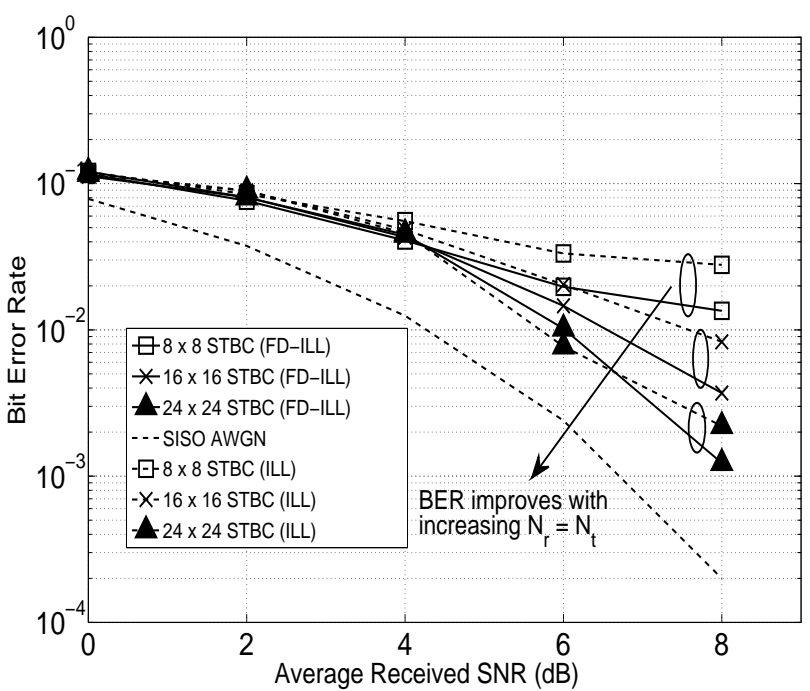

Fig. 4

UNCODED BER OF BP DECODING OF $8 \times 8,16 \times 16$ AND $24 \times 24$ NON-ORTHOGONAL STBCS. ILL $(\delta=t=1)$ AND FD-ILL

$\left(\delta=e^{\sqrt{5} \mathbf{j}}, t=e^{\mathbf{j}}\right)$ STBCS. $N_{t}=N_{r}$, BPSK, 5 BP ITERATIONS. $B P$ decoding achieves near SISO AWGN performance for large sized STBCs.

BER. This is due to the inherent ability of BP to perform well in large systems.

- With the proposed BP decoding, the BER of ILL STBC is worse than the BER of FD-ILL STBC. This performance gap between ILL and FD-ILL STBCs diminishes with increasing $N_{t}$, indicating that for large $N_{t}=N_{r}$ ILL feature of the STBC with $\delta=t=1$ is good enough.

Turbo coded BER performance: Figure 5 shows the turbo coded BER performance of the BP detector in a STBC MIMO system with BPSK and $24 \times 24$ FD-ILL STBC (i.e., $\delta=e^{\sqrt{5} \mathbf{j}}$, $t=e^{\mathbf{j}}$ ) and $N_{t}=N_{r}=24$. Rate- $1 / 3$ and rate-1/2 turbo codes achieving $8 \mathrm{bps} / \mathrm{Hz}$ and $12 \mathrm{bps} / \mathrm{Hz}$ spectral efficiencies, respectively, are used. The theoretical minimum SNRs required to achieve these 8 and $12 \mathrm{bps} / \mathrm{Hz}$ capacities in a $N_{t}=N_{r}=24$ MIMO channel, as computed from the ergodic capacity formula [1], are also shown in Fig. 5. From Fig. 5, it can be seen that the vertical fall in coded BER occurs only about $2.5 \mathrm{~dB}$ away from the theoretical minimum SNRs, which is very good in terms of nearness to capacity. Such nearness results for large STBCs from CDA using BP decoding have not been reported so far.

Effect of Spatial Correlation: In generating the BER results in Figs. 4 and 5 , we have assumed i.i.d. fading. However, MIMO propagation conditions witnessed in practice often render the i.i.d. fading model as inadequate. More realistic MIMO channel models that take into account the scattering environment, spatial correlation, etc., have been investigated in the literature [19]-[21]. For example, spatial correlation at the transmit and/or receive side can affect the rank structure of the MIMO channel resulting in degraded MIMO capacity [19]. The structure of scattering in the propagation environment can also affect the capacity [20]. Hence, it is of interest to investigate the performance of the proposed BP decoder in more realistic MIMO channel models. Towards 


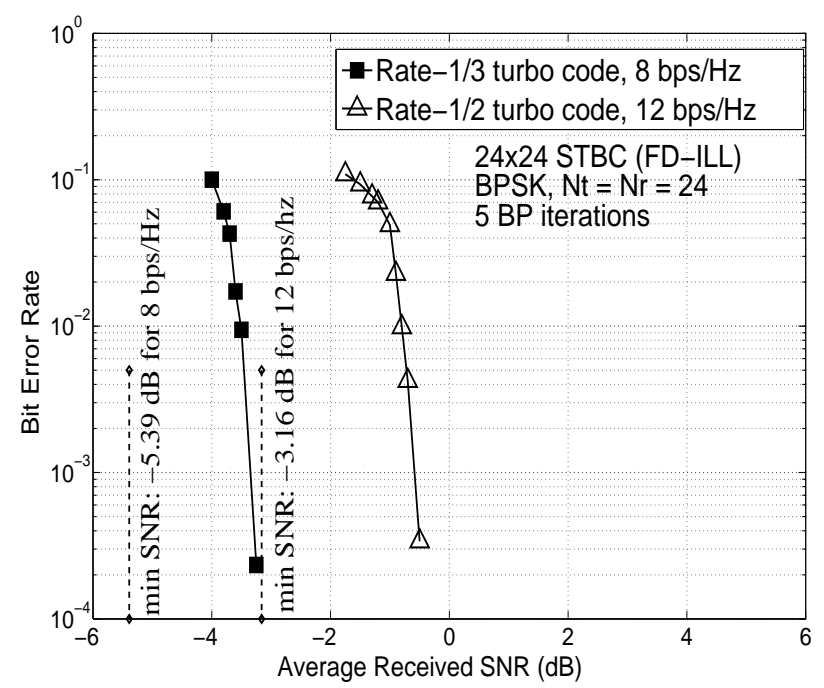

Fig. 5

TURBO CODED BER OF BP DECODING OF $24 \times 24$ NON-ORTHOGONAL FD-ILL STBC $\left(\delta=e^{\sqrt{5} \mathbf{j}}, t=e^{\mathbf{j}}\right) . N_{t}=N_{r}=24$, BPSK, TURBO CODE RATES: RATE-1/2 (12 BPS/Hz) AND RATE-1/3 (8 BPS/Hz). 5 BP ITERATIONS. BP decoding achieves near-capacity performance to within about $2.5 \mathrm{~dB}$ from capacity.

this end, in this subsection, we adopt the correlated MIMO channel model in [21], which incorporates the single spatial correlation parameter, $r$, presented in [22], to a matrix channel model. Figure 6 shows the simulated uncoded BER performance of $16 \times 16$ FD-ILL STBC with BPSK, $N_{t}=16$, $N_{r}=16,17$ for the correlation channel model in [21] with $r=0.12$. Performance of the same with i.i.d fading and $N_{t}=N_{r}=16$ is also plotted for comparison. From Fig. 6, it can be observed that compared to i.i.d fading, there is a loss in diversity order in spatial correlation for $N_{t}=N_{r}=16$; further, use of more receive antennas $\left(N_{r}=17, N_{t}=16\right)$ alleviates this loss in performance. We note that the proposed BP based decoding can be used to decode perfect codes [23], [24] of large dimensions as well.

\section{CONClusion}

We presented a low-complexity decoding scheme based on $\mathrm{BP}$ to decode non-orthogonal STBCs from CDA having large dimensions. The proposed BP scheme involved message passing on Markov random field representation of the STBC MIMO system. Successful application of BP for detection in large non-orthogonal STBC MIMO systems has not been reported so far. Simulation results showed that the BP approach to large STBC decoding is quite effective, achieving near-ML and near-capacity performance in STBCs with large dimensions. Effect of spatial correlation on the performance of the proposed BP decoding was presented. Extension of the proposed BP approach to higher order modulation (e.g., $M$ $\mathrm{PAM} / M$-QAM) in the large STBC MIMO context can be investigated as further extension to this work.

\section{REFERENCES}

[1] H. Jafarkhani, Space-Time Coding: Theory and Practice, Cambridge University Press, 2005.

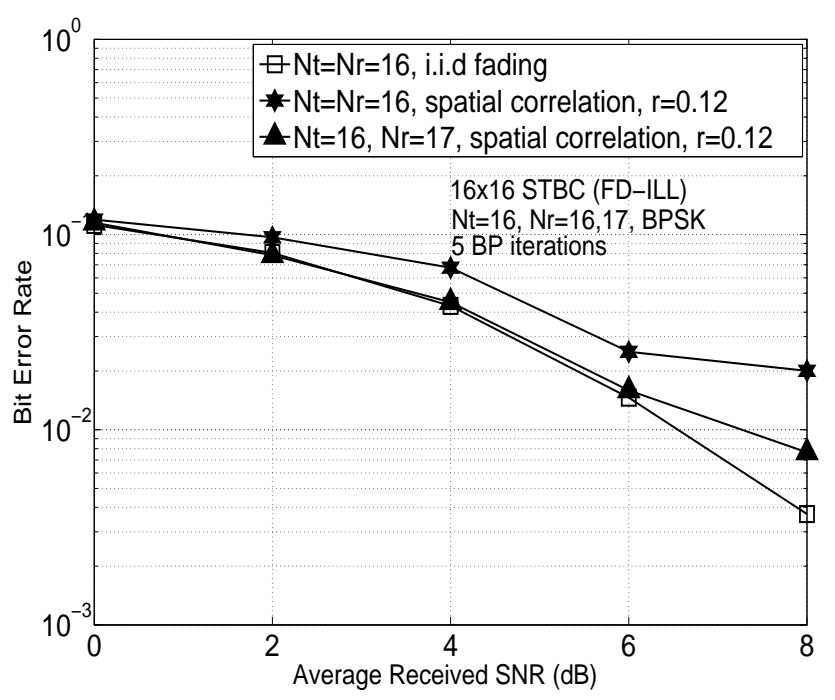

Fig. 6

EFFECT OF SPATIAL CORRELATION ON THE UNCODED BER OF BP DECODING OF $16 \times 16$ NON-ORTHOGONAL FD-ILL STBC $\left(\delta=e^{\sqrt{5} \mathbf{j}}, t=e^{\mathbf{j}}\right) . N_{t}=16, N_{r}=16,17$, BPSK, 5 BP ITERATIONS, $r=0.12$. Spatial correlation degrades achieved diversity order compared to that achieved in i.i.d fading. Increasing $N_{r}$ alleviates this performance loss.

[2] B. A. Sethuraman, B. Sundar Rajan, and V. Shashidhar, "Full-diversity high-rate space-time block codes from division algebras," IEEE Trans. Inform. Theory, vol. 49, no. 10, pp. 2596-2616, October 2003.

[3] F. Oggier, J.-C. Belfiore, and E. Viterbo, Cyclic Division Algebras: A Tool for Space-Time Coding, Foundations and Trends in Commun. and Inform. Theory, vol. 4, no. 1, pp. 1-95, Now Publishers, 2007.

[4] J.-C. Belfiore, G. Rekaya, and E. Viterbo, "The golden code: A $2 \times$ 2 full-rate space-time code with non-vanishing determinants," IEEE Trans. Inform. Theory, vol. 51, no. 4, April 2005.

[5] K. Vishnu Vardhan, Saif K. Mohammed, A. Chockalingam, B. Sundar Rajan, "A low-complexity detector for large MIMO systems and multicarrier CDMA systems," IEEE JSAC Spl. Iss. on Multiuser Detection, for Adv. Commun. Systems and Networks, pp. 473-485, April 2008.

[6] Saif K. Mohammed, A. Chockalingam, and B. Sundar Rajan, "A lowcomplexity near-ML performance achieving algorithm for large MIMO detection," Proc. IEEE ISIT'2008, Toronto, July 2008.

[7] Saif K. Mohammed, A. Chockalingam, and B. Sundar Rajan, "Highrate space-time coded large MIMO systems: Low-complexity detection and performance," Proc. IEEE GLOBECOM'2008, December 2008.

[8] J. S. Yedidia, W. T. Freeman, Y. Weiss, "Understanding belief propagation and its generalizations," MERL Tech Rep. TR-2001-22, Jan. 2002.

[9] B. J. Frey, Graphical Models for Machine Learning and Digital Communication, Cambridge: MIT Press, 1998.

[10] R. J. McEliece and D. J. C. MacKay, and J-F. Cheng, "Turbo decoding as an instance of Pearl's belief propagation algorithm," IEEE Jl. Sel. Areas in Commun., vol. 16, no.2, pp. 140-152, February 1998.

[11] D. J. C. MacKay, "Good error-correcting codes based on very sparse matrices," IEEE Trans. Inform. Theory, pp. 399-431, March 1999.

[12] A. Montanari, B. Prabhakar, and D. Tse, "Belief propagation based multiuser detection," Online arXiv:cs/0510044 2 [cs.IT] 22 May 2006.

[13] D. Guo and C-C. Wang, "Multiuser detection of sparsely spread CDMA," IEEE JSAC Spl. Iss. on Multiuser Detection, for Adv. Commun. Systems and Networks, vol. 26, no. 3, pp. 421-431, April 2008.

[14] O. Shental, A. J. Weiss, N. Shental, Y. Weiss, "Generalized belief propagation receiver for near-optimal detection of two-dimensional channels with memory," IEEE Inform. Theory Workshop, October 2004.

[15] G. Colavolpe and G. Germi, "On the application of factor graphs and the sum-product algorithm to ISI channels," IEEE Trans. on Commun., vol. 53, no. 5, pp. 818-825, May 2005.

[16] J. Soler-Garrido, R. J. Piechocki, K. Maharatna, and D. McNamara, "Analog MIMO detection on the basis of belief propagation," Proc. IEEE Mid-West Symp. on Circuits and Systems, 2006.

[17] X. Yang, Y. Xiong, F. Wang, "An adaptive MIMO system based on unified belief propagation detection," Proc. IEEE ICC'2007, June 2007. 
[18] K. Murphy, Y. Weiss, and M. Jordan, "Loopy belief propagation for approximate inference: An empirical study," 15th Annual Conf. on Uncertainty in Artificial Intelligence, pp. 467-470, 1999.

[19] D. Shiu, G. J. Foschini, M. J. Gans, J. M. Khan, "Fading correlation and its effect on the capacity of multi-antenna systems," IEEE Trans. Commun., vol. 48, pp. 502-513, March 2000.

[20] D. Gesbert, H. Bölcskei, D. A. Gore, A. J. Paulraj, "Outdoor MIMO wireless channels: Models and performance prediction," IEEE Trans. on Commun., vol. 50, pp. 1926-1934, December 2002.

[21] A. van Zelst and J.S. Hammerschmidt, "A single coefficient spatial correlation model for multiple-input multiple-output (MIMO) radio channels," 27th General Assembly of the Intl. Union of Radio Science (URSI), Maastricht, Netherlands, August 2002.

[22] G. D. Durgin and T. S. Rappaport, "Effects of multipath angular spread on the spatial correlation of received voltage envelopes," Proc. Proc. IEEE VTC'1999, pp. 996-1000, 1999.

[23] F. E. Oggier, G. Rekaya, J.-C. Belfiore, and E. Viterbo, "Perfect spacetime block codes," IEEE Trans. on Inform. Theory, vol. 52, no. 9, September 2006.

[24] P. Elia, B. A. Sethuraman, and P. V. Kumar, "Perfect space-time codes for any number of antennas," IEEE Trans. Inform. Theory, vol. 53, no. 11, pp. 3853-3868, November 2007. 